\title{
ARTICLE
}

\section{Structural-functional decoupling predicts suicide attempts in bipolar disorder patients with a current major depressive episode}

\author{
Haiteng Jiang ${ }^{1}$, Rongxin Zhu ${ }^{1}$, Shui Tian ${ }^{2,3}$, Huan Wang ${ }^{2,3}$, Zhilu Chen ${ }^{1}$, Xinyi Wang ${ }^{2,3}$, Junneng Shao ${ }^{2,3}$, Jiaolong Qin ${ }^{4}$, Jiabo Shi ${ }^{1}$, \\ Haiyan Liu', Yu Chen ${ }^{1}$, Zhijian Yao ${ }^{1,5}$ and Qing Lu iD $^{2,3}$
}

Bipolar disorder (BD) is associated with a high risk of suicidality, and it is challenging to predict suicide attempts in clinical practice to date. Although structural and functional connectivity alterations from neuroimaging studies have been previously reported in BD with suicide attempts, little is known about how abnormal structural and functional connectivity relates to each other. Here, we hypothesize that structure connectivity constrains functional connectivity, and structural-functional coupling is a more sensitive biomarker to detect subtle brain abnormalities than any single modality in BD patients with a current major depressive episode who had attempted suicide. By investigating structural and resting-state fMRI connectivity, as well as their coupling among 191 BD depression patients with or without a history of suicide attempts and 113 healthy controls, we found that suicide attempters in BD depression patients showed significantly decreased central-temporal structural connectivity, increased frontal-temporal functional connectivity, along with decreased structural-functional coupling compared with non-suicide attempters. Crucially, the altered structural connectivity network predicted the abnormal functional connectivity network profile, and the structural-functional coupling was significantly correlated with suicide risk but not with depression or anxiety severity. Our findings suggest that the structural connectome is the key determinant of brain dysfunction, and structural-functional coupling could serve as a valuable trait-like biomarker for BD suicidal predication over and above the intramodality network connectivity. Such a measure can have clinical implications for early identification of suicide attempters with BD depression and inform strategies for prevention.

Neuropsychopharmacology (2020) 45:1735-1742; https://doi.org/10.1038/s41386-020-0753-5

\section{INTRODUCTION}

Bipolar disorder (BD), typically characterized by recurrent episodes of mania/hypomania and depression, is a mental disorder most associated with suicide, especially during the major depressive episode [1, 2]. Based on the statistic, the estimated prevalence of suicide attempts in BD type I and BD type II was about $32.4 \%$ and $36.3 \%$, respectively [3], which is $\sim 20-30$-fold greater than the general population [1]. Given the high suicide risk in $B D$, early identification and intervention are particularly important in clinical practice. However, the current suicide risk identification, which mainly relies on clinical assessments such as interviews or scale, is unsatisfactory because of the subjectivity of retrospective clinical information and self-reports by patients. Besides, about $80 \%$ of persons who committed suicide would cover up their suicidal ideation to doctors or health providers [4]. Therefore, objective neural markers are in urgent need to identify and predict suicide attempts as early as possible.

The advent of brain imaging techniques such as diffusion tensor imaging (DTI) and functional magnetic resonance imaging (fMRI) provide the new avenue to probe the structurally and functionally interconnected brain network [5-8]. Structural connectivity (SC) can be mapped via DTI using white matter tractography, while functional connectivity (FC) can be quantified by the temporal correlation between blood oxygen level-dependent fMRI signals. Abnormalities in SC and FC have been reported in BD with attempted suicide behaviors [9-12]. However, most previous studies in suicidal behavior focus on only a single modality, which might be insufficient to depict the pathological changes $[13,14]$. Therefore, the combination of both structural and FC may help better understand the pathological mechanism of suicide behaviors.

Structural and functional connectivity provides different perspectives on brain function, and they are interrelated. It has been suggested that SC shapes and constrains FC across the brain network at various spatial scales, while FC exerts influences on SC through the plasticity mechanism $[5,15]$. As such, SC-FC coupling was proposed to study the association between SC and FC. Studies have demonstrated SC-FC coupling's potential to detect

\footnotetext{
${ }^{1}$ Department of Psychiatry, The Affiliated Brain Hospital of Nanjing Medical University, 264 Guangzhou Road, Nanjing 210029, China; ${ }^{2}$ School of Biological Sciences and Medical Engineering, Southeast University, 2 Sipailou Street, Nanjing 210096, China; ${ }^{3}$ Child Development and Learning Science, Key Laboratory of Ministry of Education, Southeast University, Nanjing 210096, China; ${ }^{4}$ Key Laboratory of Intelligent Perception and Systems for High-Dimensional Information of Ministry of Education, School of Computer Science and Engineering, Nanjing University of Science and Technology, Nanjing 210094, China and ${ }^{5}$ Nanjing Brain Hospital, Medical School of Nanjing University, 22 Hankou Road, Nanjing 210093, China
}

Correspondence: Zhijian Yao (zjyao@njmu.edu.cn) or Qing Lu (luq@seu.edu.cn)

These authors contributed equally: Haiteng Jiang, Rongxin Zhu

Received: 10 April 2020 Revised: 14 June 2020 Accepted: 22 June 2020

Published online: 30 June 2020 
more subtle brain abnormalities than any single modality $[13,14,16,17]$. However, it is still unknown whether SC-FC coupling is altered in $\mathrm{BD}$ depression patients with suicide attempts and how abnormal SC and FC relates to each other.

In this study, we recruited a large cohort of participants $(N=$ 304, 191 BD depression patients with or without a history of suicide attempts and 113 healthy controls) and combined structural and FC to investigate the neuropathology related to suicide attempts in BD depression patients. Since SC provides the physical basis for information flow in the brain [18], we predict that abnormal SC network should impose strong constraints on FC network. Furthermore, we hypothesize that SC-FC coupling may provide a more sensitive biomarker to detect subtle brain abnormalities than SC or FC, and may shed new insights into the understanding of the pathophysiological mechanisms in BD depression patients with suicide attempts.

\section{MATERIALS AND METHODS}

\section{Participants}

A total of 191 in patients diagnosed with BD were recruited from the Affiliated Brain Hospital of Nanjing Medical University between September 2014 and April 2019. All BD patients were in the major depressive episode and diagnosed by at least two attending psychiatrists using the Mini-International Neuropsychiatric Interview (MINI, Chinese version) [19], according to the criteria of the Diagnostic and Statistical Manual of Mental Disorders, 4th Edition, Text Revision (DSM-IV-TR). The inclusion criteria were as follows: (1) right-handed and native Han Chinese; (2) age between 18 and 55 years; (3) the 17-item Hamilton Depression Rating Scale (HAMD-17) scores $\geq 17$; (4) no comorbidity with other mental disorders that meet criteria of DSM-IV axis-I disorders; (5) no systematic psychotherapy or physical therapy in the past 6 months; (6) no psychotropic medication use including antidepressants, mood stabilizers, and antipsychotics in the past 6 weeks. For healthy controls (HC), 113 participants who had no lifetime DSM-IV axis-I psychiatric disorders, a history of substance abuse or dependence assessed by MINI, no family history of any mental disorders among their first-degree relatives assessed by Family History Screen for Epidemiological Studies [20], and no history of neurological disorders were recruited from the local community. Besides, participants were excluded if they had the following conditions: (1) severe somatic diseases and serious neurological disorders confirmed by the past medical history or laboratory test; (2) pregnant or active breastfeeding female participants; (3) inability to undergo magnetic resonance imaging (MRI) scan.

All participants underwent an MRI scan within 2 days of the clinical examination. For all BD depression patients, the MRI scanning was always conducted prior to the initiation of treatment. Among them, $15 \mathrm{BD}$ and $1 \mathrm{HC}$ participants were excluded due to excessive head movements and poor image quality. In the final analyses, $176 \mathrm{BD}$ and $112 \mathrm{HC}$ were included. Furthermore, the suicide attempt was evaluated according to the definition by the American Psychiatric Association in 2003. Suicide attempt was defined if participants had at least one documented self-injurious act with the intent to die in the current episode, confirmed by medical records and the HAMD-17 3-item (suicide) score $\geq 2$ [21]. Subsequently, BD depression patients were divided into two subgroups: 75 individuals with at least one suicide attempt during the recent depressive episode (SA group) and 101 with no suicide attempt history during the present or in previous depressive episodes (NSA group).

\section{Ethical statement}

This study was approved by the Local Medical Ethics Committee at the Affiliated Brain Hospital of Nanjing Medical University and abided by the ethical guidelines of the World Medical Association
Declaration of Helsinki [22]. Written informed consent was provided to all participants.

\section{Clinical assessments}

Age, gender, and education information were collected for all participants. Among BD patients, the severity of depressive symptoms and anxiety symptoms were assessed using the 17item HAMD [23] and the Hamilton Anxiety Rating Scale (HAMA) [24], respectively. In addition, the suicide risk in BD was assessed using the Nurses' Global Assessment of Suicide Risk (NGASR) [25]. The NGASR is an "evidence-based" suicide risk evaluation scale, including not only current clinical manifestations (e.g., presence/ influence of hopelessness) but also distal clinical factors (e.g., family history of serious psychiatric problems or suicide). Fifteen factors are involved in the scale with different weightings (see Supplementary Table S1). The total NGASR score represents the severity of suicide risk, thus the higher score corresponds to higher suicide risk. Table 1 summarized the demographic and clinical characteristics of all participants.

Neuroimaging data acquisitions and preprocessing

MRI data acquisition. The fMRI data of all participants were acquired using a 3.0 T Siemens MRI system (Siemens Medical Solutions, Germany) at the Department of Medical Imaging, the Affiliated Brain Hospital of Nanjing Medical University. The DTI and resting-state functional data were collected while participants were instructed to keep their eyes closed, minimize head movement, stay awake, and not think about anything. No participants reported falling asleep during the scanning when asked right after the scanning. Head motion was limited to $<2.5 \mathrm{~mm}$ translation and/or $2.5^{\circ}$ rotation.

The DTI data were obtained with the following parameters: diffusion was measured along 30 noncollinear directions with $b=1000 \mathrm{~s} / \mathrm{mm}^{2}$, and an additional image without diffusion weighting with $b=0$, repetition time (TR) $=6600 \mathrm{~ms}$, echo time $(\mathrm{TE})=93 \mathrm{~ms}$, flip angle $=90^{\circ}$, matrix $=128 \times 128$, field of view $(F O V)=240 \times 240 \mathrm{~mm}^{2}$, slice thickness $/$ gap $=3 / 0 \mathrm{~mm}$, acquisition voxel size $=1.875 \times 1.875 \times 3 \mathrm{~mm}^{3}$. The resting-state functional data were obtained using an echo-planar imaging sequence with the following parameters: $T R=3000 \mathrm{~ms}$, TE $=$ $40 \mathrm{~ms}$, matrix $=64 \times 64, \mathrm{FOV}=240 \times 240 \mathrm{~mm}^{2}$, flip angle $(\mathrm{FA})=$ $90^{\circ}$, voxel size $=3.75 \times 3.75 \times 4 \mathrm{~mm}^{3}, 32$ axial slices with thickness $/ g a p=4 / 0 \mathrm{~mm}$, and 133 volumes. For the T1-weighted image, the acquisition parameters were as follows: TR $=1900$ $\mathrm{ms}, \mathrm{TE}=2.48 \mathrm{~ms}$, matrix $=256 \times 256, \quad F O V=250 \times 250 \mathrm{~mm}^{2}$, thickness $/$ gap $=1.0 / 0 \mathrm{~mm}$, flip angle $=9^{\circ}$, inversion time $=900$ $\mathrm{ms}$, and acquisition voxel size $=1 \times 1 \times 1 \mathrm{~mm}^{3}$.

Structural connectivity preprocessing. The DTI data were preprocessed using the FMRIB Software Library toolbox [26]. The head motion artifacts and eddy current distortions were corrected by applying a rigid-body transformation to the b0 image. Then, the diffusion tensor matrix was computed based on the Stejskal and Tanner equation. By diagonalizing the tensor matrix, three eigenvalues and eigenvectors were acquired, and FA maps were estimated. Following that, we registered each b0 image to the Montreal Neurological Institute (MNI) space via the corresponding T1 image with Diffusionkit [27]. For the fiber tracking, diffusion toolkit was used, and whole-brain tractography was computed via fiber assignment by continuous tracking algorithm [28]. Fiber tracking was terminated when $\mathrm{FA}<0.2$ or the minimum angle was larger than $50^{\circ}$.

Resting-state functional connectivity preprocessing. The restingstate fMRI data were preprocessed by the Data Processing Assistant for Resting-State fMRI [29] and the Statistical Parametric Mapping software (SPM8; http://www.fil.ion.ucl.ac.uk/spm). The first six volumes were excluded to allow signal equilibrium. Then, 
Table 1. Demographic and clinical characteristics of the sample.

\begin{tabular}{|c|c|c|c|c|c|}
\hline Characteristic & SA & NSA & Controls & $t / F / x^{2}$ & $P$ \\
\hline Age (years) & $27.11 \pm 9.00$ & $31.00 \pm 10.35$ & $32.65 \pm 9.28$ & $7.61^{\mathrm{a}}$ & $0.001^{* *}$ \\
\hline Gender (male/female) & $22 / 53$ & $43 / 58$ & $41 / 71$ & $3.25^{\mathrm{c}}$ & 0.197 \\
\hline Course of disease (months) & $63.32 \pm 71.62$ & $73.79 \pm 66.81$ & & $-0.98^{b}$ & 0.329 \\
\hline HAMD-17 (3rd item, suicide) & $3.12 \pm 0.62$ & $1.63 \pm 1.10$ & & $11.4^{\mathrm{b \#}}$ & $0.000^{* * *}$ \\
\hline Total scores (HAMA) & $15.47 \pm 6.87$ & $16.98 \pm 7.84$ & & $-1.32^{\mathrm{b \#}}$ & 0.189 \\
\hline Total scores (NGASR) & $13.16 \pm 2.32$ & $7.25 \pm 2.99$ & & $14.67^{\mathrm{b \#}}$ & $0.000^{* * *}$ \\
\hline Number of episodes of depression & $3.60 \pm 2.38$ & $3.10 \pm 1.81$ & & $1.55^{\mathrm{b \#}}$ & 0.124 \\
\hline Number of episodes of mania/hypomania & $2.29 \pm 2.47$ & $2.29 \pm 1.89$ & & $-0.02^{\mathrm{b} \#}$ & 0.983 \\
\hline
\end{tabular}

the remaining volumes were correlated for slice timing, realigned for head motion corrections, and spatially normalized to the MNI space. After that, the normalized images were resampled to $3 \times$ $3 \times 3 \mathrm{~mm}^{3}$, smoothed with $6 \mathrm{~mm}$ kernel and band-pass filtered with $0.01-0.08 \mathrm{~Hz}$ to reduce low-frequency drift effects and highfrequency noise. Source of spurious variances (e.g., Friston 24 parameter obtained via head motion correction, the signals from the whole brain, white matter, and cerebrospinal fluid) were removed through linear regression. Participants were excluded if their head motions exceed $2.5 \mathrm{~mm}$ translationally or $2^{\circ}$ in any direction.

Structural connectivity network and functional connectivity network construction. We defined each region of interest from automated anatomical labeling (AAL) atlas consisting of 90 regions as a network node (see Supplementary Table S2) [30]. Using inverse transformations, AAL atlas from the MNI space was registered to the individual's native space. In native diffusion space, region (i) and region (j) were considered structurally connected through an edge $e=(\mathrm{i}, \mathrm{j})$ if the length of passing fibers were longer than $10 \mathrm{~mm}$ and there were at least two streamline counts. For each edge, we computed the mean FA values of all fibers as its weights.

To obtain the FC network, the averaged fMRI time series were extracted from each of the AAL atlas with 90 regions. Then, Pearson correlations were computed on the averaged time series between all paired regions, resulting in a $90 \times 90 \mathrm{FC}$ matrix. To improve the normality, Fisher- $Z$ transformation was applied to the correlation coefficients.

Structural-functional coupling analysis. SC-FC coupling was estimated by the correlation between the strengths of structural and FC networks [15]. For each participant, all nonzero SC edges were selected from the SC network and rescaled into a Gaussian distribution. Then, their corresponding FC from the FC network was extracted and correlated with their structural counterparts. This results in a single structural-functional coupling metric for each participant, representing the SC-FC coupling strength within the network.
Statistical analysis. The statistics of demographic and clinical characteristics were conducted using Statistical Product and Service Solutions (SPSS, version 22.0). For statistics between three groups, $x^{2}$ tests were used to compare gender. Age and education were analyzed using a one-way analysis of variance. When comparing between SA and NSA groups, $X^{2}$ tests were conducted to compare the family history of suicide and comorbid substance abuse/dependence; two-sample $t$ tests were used to compare HAMD-17, HAMA, number of previous episodes, and NGASR. $P<$ 0.05 was considered statistically significant.

The network-based statistic (NBS) was used to evaluate the significant statistical network between different groups [31]. NBS is a method based on the principles of classical cluster-based thresholding of statistical parametric maps to control the familywise error rate when mass-univariate testing is performed at every connection comprising the graph. When comparing the structural and functional network differences between SA, NSA, and HC, an independent $F$ test was conducted on every connectivity value. Connections with $F$ values exceeding the primary threshold were selected to form the topological clusters, in which the number of connections within each cluster was defined as the observed cluster score. Note that the choice of initial cluster-forming threshold (primary threshold) depends on the researchers' arbitrary decision, and there is no widely accepted criterion. Here, we followed the recent NBS studies and set the primary threshold at the $p=0.001$ level to have a stringent control of Type I error $[32,33]$. Next, the data across groups were randomized 5000 times to obtain the reference cluster distribution. For each randomization, we used the maximum number of connections across all clusters to form the reference distribution. Then, observed cluster scores exceeding the 95th percentile were considered as significant $(P<0.05)$. For the post hoc paired comparisons (e.g., SA vs. NSA), the procedure was similar. The differences were described as follows. We first masked the network connectivity matrix with identified significant connections among three groups and then applied an independent two-sample $t$ test on the connectivity value. Note that age and education were entered as nuisance variables during the NBS since they were significantly different between groups. 


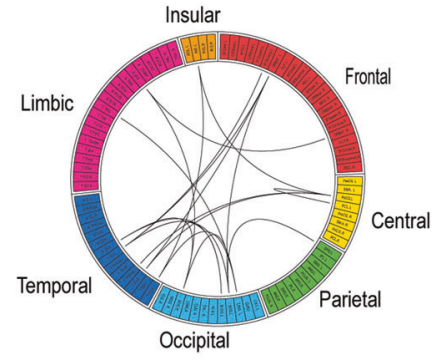

B

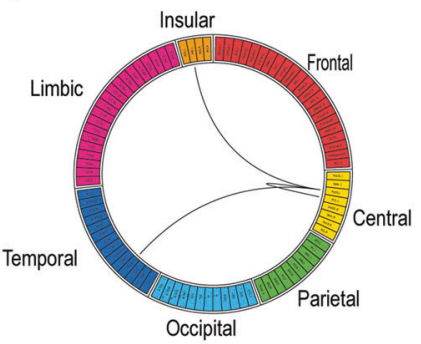

SA vs. NSA

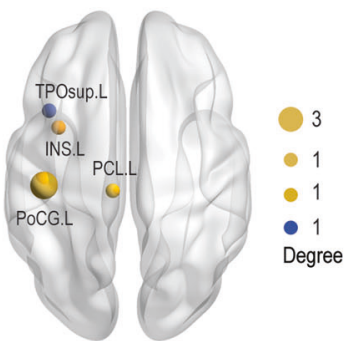

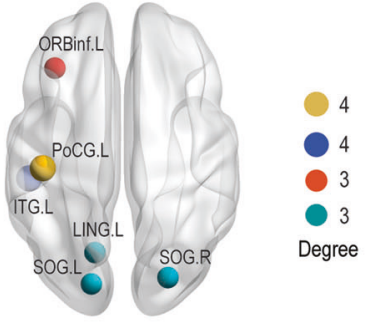

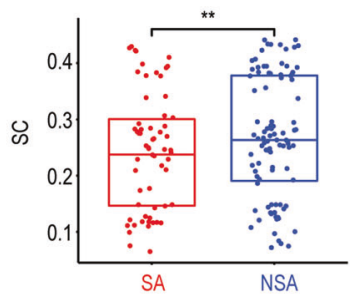

Fig. 1 Structural connectivity (SC) differences. a The structural connectome network differences between SA, NSA, and HC identified by NBS. A link in the circular plot represents a significantly different connection. The most involved nodes (degree $\geq 3$ ) are shown on the right panel, in which the size of the node corresponds to the number of significant connections. $\mathbf{b}$ Similar to a but between SA and NSA. Bar plots showing mean connectivity obtained within the significant network for SA and NSA. There was a significant difference between these two groups $(p<0.01) .{ }^{* *} P<0.01$. SA suicide attempt, NSA non-suicide attempt, HC healthy controls.

\section{RESULTS}

Demographic and clinical characteristics

The demographic and clinical information of all participants is summarized in Table 1 . The three groups were significantly different in age and education (age: $F_{(2,285)}=7.61, P<0.001$; education: $\left.F_{(2,285)}=9.31, P<0.0001\right)$. The SA group scored significantly higher on the 3rd item of HAMD in suicide and NGASR compared to the NSA group $(P<0.0001)$. Since age and education were significantly among three groups, they were treated as nuisance variables and controlled in all subsequent neural data analysis.

\section{Structural connectivity network}

First, we examined the SC network differences between SA, NSA, and HC. NBS revealed a significantly altered central and temporal network (Fig. 1a). This network consisted of 18 regions and 19 connections, mainly involving left postcentral gyrus, left inferior temporal gyrus, left inferior frontal gyrus (orbital part), left lingual gyrus, and bilateral superior occipital gyrus. The post hoc analysis between SA and NSA showed a decreased central-temporal SC network in SA compared with NSA, which was composed of four regions (left postcentral gyrus, left insula, left paracentral lobule, and left superior temporal gyrus) and three connections (Fig. 1b). More specifically, the left postcentral gyrus was the hub of the altered SC network.

Functional connectivity network

Next, we investigated the FC network differences between SA, NSA, and $\mathrm{HC}$. There was a significantly changed frontal-temporal network identified by NBS (Fig. 2a). This network included 39 regions and 48 connections, predominantly containing right inferior frontal gyrus (orbital part), left superior temporal gyrus, right middle frontal gyrus (orbital part), left inferior frontal gyrus (opercular part), right superior parietal gyrus, and left insular. Subsequently, the post hoc analysis between SA and NSA showed a significantly increased frontal-temporal FC network (Fig. 2b). This altered FC network compromised seven regions and six connections, predominantly involving right middle temporal gyrus, left precentral gyrus, right inferior frontal gyrus (triangular part), and right fusiform gyrus.

Structural-functional connectivity relations

After identifying the abnormal SC and FC network, we then ask how they are related to each other. A cross-modality analysis was conducted, in which we evaluated the SC in the altered FC network (Fig. 1a) and the FC in the altered SC network (Fig. 2a). Interestingly, we found that the FC within the altered SC network was significantly different between three groups $\left(F_{(2,285)}=4.25\right.$, $P<0.05$, Fig. 3a), while this was not the case in the SC within the abnormal FC network $\left(F_{(2,285)}=0.83, P>0.4\right.$, Fig. 3b). Moreover, structural-functional connectivity coupling was investigated at both the whole-brain level and local networks by combing the altered SC and FC network in Figs. 1a, 2a. The structural-functional connectivity couplings were significantly different between the three groups at both the whole-brain network $\left(F_{(2,285)}=11.37, P<\right.$ 0.001 , Fig. 3c) and the altered subnetwork $\left(F_{(2,285)}=5.25, P<0.05\right.$, Fig. 3d). However, significant structural-functional decoupling difference between SA and NSA was only observed in the combined abnormal SC and FC networks $(t(174)=4.84, P<0.001$, Fig. 3d).

Suicide risk and neuropsychological correlates

Finally, correlational analyses were performed between SC, FC, and SC-FC coupling in which SA and NSA group differences were detected. Relationships of SC, FC, and SC-FC coupling abnormalities to clinical assessments (suicide risk, depression and anxiety severity) were evaluated using Spearman correlation. There were no associations between NGASR and SC within the altered SC network or FC within the altered FC network. However, we did find a significant negative correlation between SC-FC coupling within the altered SC and FC network and NGASR (Fig. 4a, $r=-0.32, p<$ $0.001)$. Importantly, the identified abnormal SC-FC couplings were not significantly correlated with the severity of depression (Fig. $4 \mathrm{~b}$, $r=-0.12, p>0.2$ ) or anxiety (Fig. $4 c, r=-0.01, p>0.8$ ). 
A

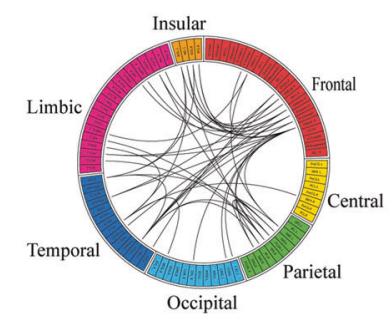

SA vs. NSA vs. HC

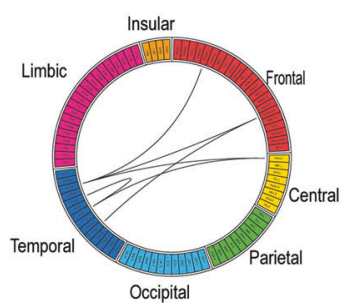

SA vs. NSA

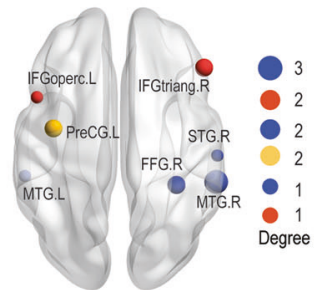

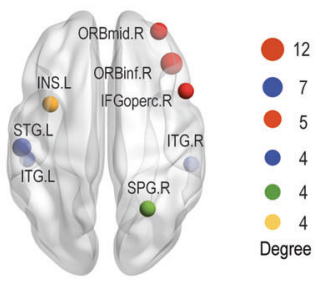

Fig. 2 Functional connectivity (FC) differences. a The functional connectome network differences between SA, NSA, and HC identified by NBS. A link in the circular plot represents a significantly different connection. The most involved nodes (degree $\geq 4$ ) are shown on the right panel, in which the size of the node corresponds to the number of significant connections. $\mathbf{b}$ Similar to a but between SA and NSA. Bar plots showing mean connectivity obtained within the significant network for SA and NSA. There was a significant difference between these two groups $(p<0.005)$. SA suicide attempt, NSA non-suicide attempt, HC healthy controls. ${ }^{* *} P<0.005$.

A $\quad$ FC within altered SC network

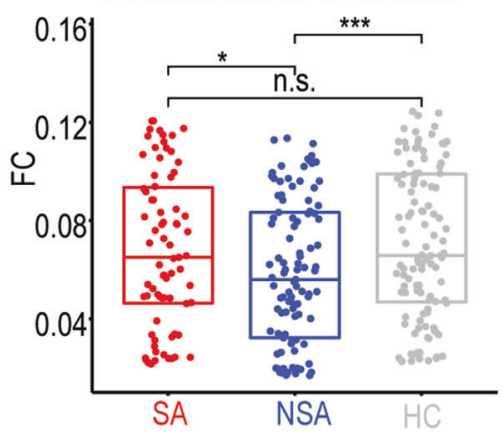

C

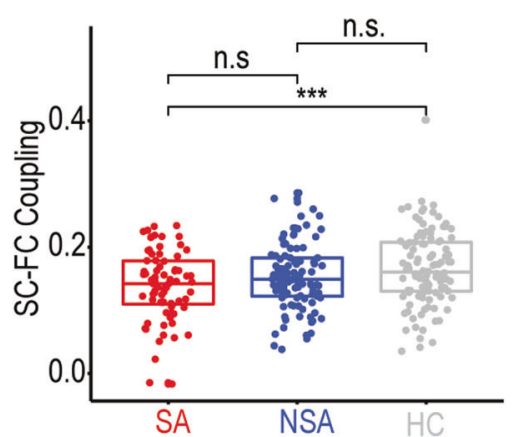

B SC within altered FC network

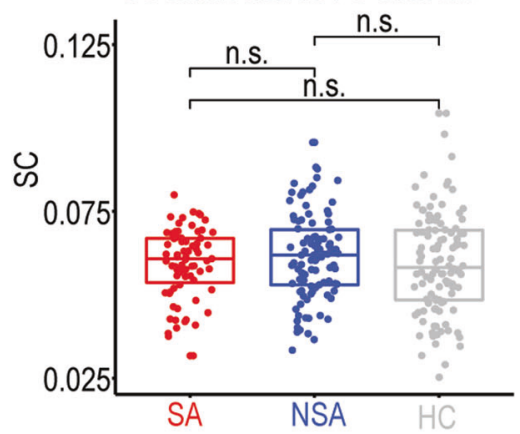

D

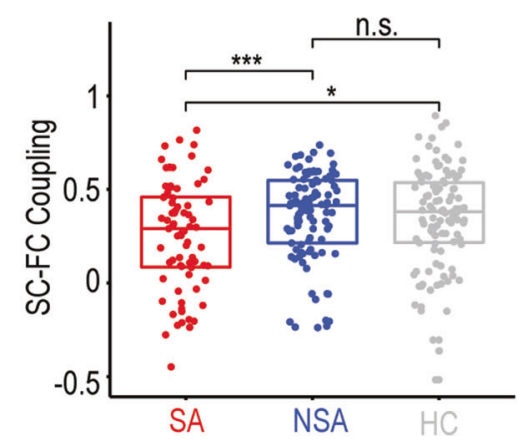

Fig. 3 Structural-functional connectivity relations. a Mean FC within the significantly altered SC network. b Mean SC within the significantly altered FC network. c Structural-functional coupling differences between SA, NSA, and HC at the whole-brain network. d Similar to c but in the significantly altered SC and FC network. SA suicide attempt, NSA non-suicide attempt, HC healthy controls, SC structural connectivity, FC functional connectivity, ns not significant. ${ }^{*} p<0.05 ;{ }^{* *} p<0.005$.

\section{DISCUSSION}

In this study, we investigated the underlying neuropathological mechanisms involved in suicide attempts among BD depression patients by integrating DTI and resting-state fMRI with a large cohort of participants. Compared with the NSA group, the SA group exhibited significantly decreased central-temporal SC, increased frontal-temporal FC, as well as decreased structural-functional couplings. Furthermore, SC-FC coupling 
A

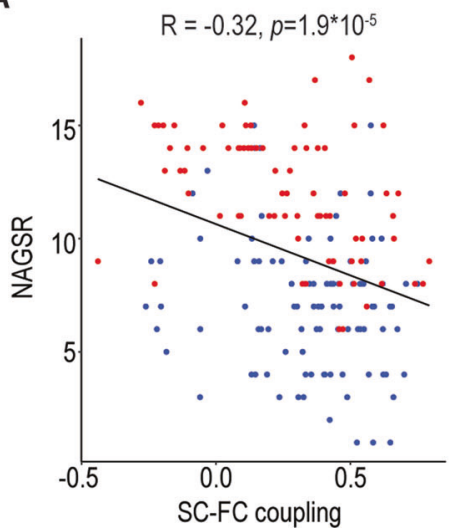

B

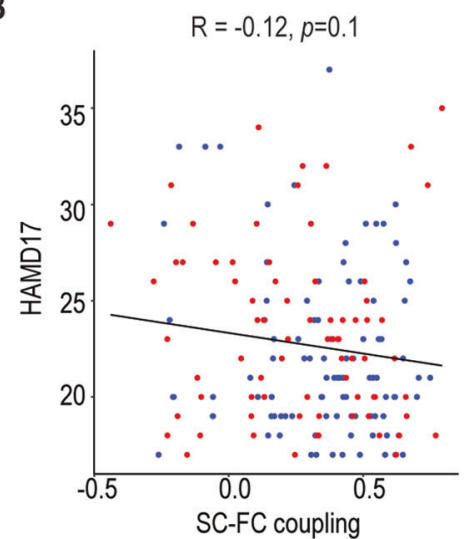

C

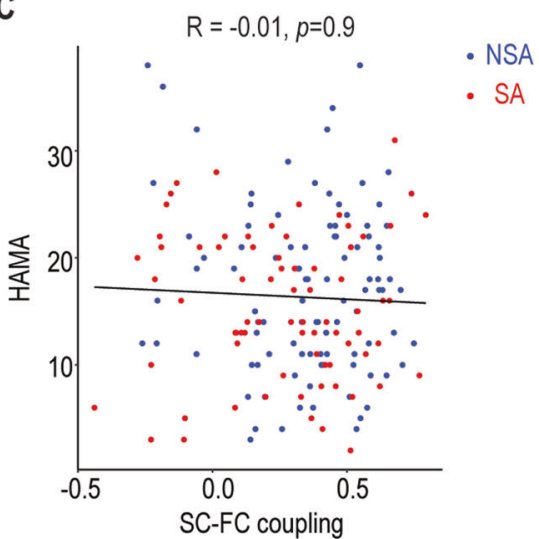

Fig. 4 Correlation between SC-FC coupling and clinical characteristics. a Correlation with suicide risk assessed by NAGSR. b Correlation with depression severity assessed by HAMD. c Correlation with anxiety assessed by HAMA. SA suicide attempt, NSA non-suicide attempt, NAGSR Nurses' Global Assessment of Suicide Risk, HAMD Hamilton Depression Rating Scale, HAMA Hamilton Anxiety Rating Scale.

was negatively correlated with the suicide risk but not with depression or anxiety severity. These findings suggested that the structural-functional decoupling was the core neuropathology involved in attempted suicide behavior among BD depression patients and could be more valuable for early identification of suicide risk than individual SC or FC modality alone.

Numerous neuroimaging studies have reported dysfunctional pathways of neural circuity involved in suicide ideation and behavior [9, 10, 34-36]. The most consistent findings across different psychiatric disorders for structural and functional alterations associated with suicide ideation and behavior converge particularly brain regions that subserve emotion and impulse regulation involving the prefrontal cortex, insular, and their mesial temporal, striatal and posterior connections, as well as in the connections between these brain areas [37]. In line with previous studies, we found decreased central-temporal SC and increased frontal-temporal and central-temporal FC in SA compared with NSA. Frontal alterations are thought to contribute to dysfunction in executive control of mood [38] and emotional pain processing [39], probably related to suicide [40, 41]. Central regions are critical for inhibitory control [42], and impairments in inhibitory control may lead to a great propensity to act on aggressive or suicidal feelings [43]. Moreover, patients with SA exhibited enhanced right middle temporal gyrus activation during emotional face task state [44] and recalled suicidal episodes [45]. Overall, our study suggests that structural and functional alterations of frontal-temporal and central-temporal networks may play a distinct role in the vulnerability for suicide behaviors in BD depression patients.

Structural and functional connectivity provide different perspectives of brain functioning, and abnormalities in both modalities have been reported in patients with suicidal behaviors and high-risk populations [9, 10, 46, 47]. However, the lack of multimodal neuroimaging studies in suicidal behaviors using both structural and functional MRI makes it challenging to understand the pathophysiological processes fully. It has been demonstrated that SC-FC coupling was informative and found to be decreased in diseases such as epilepsy [14], stroke [17], Alzheimer's disease [16], or euthymic BD [13]. Here, we investigated the SC-FC coupling for the first time in $\mathrm{BD}$ depression patients with suicide attempts. Compared with NSA, SA showed a significantly decreased SC-FC coupling at the altered SC and FC network level, suggesting a loss of coherence of structural and functional connectomes [48, 49]. Note that the significance was not preserved at the whole-brain level, indicating that confined network properties may be more sensitive than whole-brain in reflecting brain abnormalities [50-52]. Moreover, SC-FC coupling rather than the altered SC or FC was found to be negatively correlated with suicide risk (Fig. $4 a$ ), revealing a trait-like suicidal marker. Critically, SC-FC coupling was not associated with depression or anxiety (Fig. 4b, c), ruling out the potential depression cofounding factors. Overall, these findings suggest that the SC-FC coupling was a more robust suicidal predictor than $\mathrm{SC}$ or FC.

Another important finding of this study was about the directionality of suicide-related changes in SC and FC measures. That is how SC and FC abnormalities relate to each other. It has been shown that FC influences SC through mechanisms of plasticity and is constrained by the underlying SC $[5,15]$. Moreover, it has also been proved that SC predicts FC of the individual brain, and one of the dominant predictors of structure-function relations is the weights of the structure links [53]. Interestingly, there was no overlap between the altered SC network (Fig. 1a) and FC network (Fig. 2a) in our study, indicating SC and FC alterations were distinct and separate. However, we found that FC within the altered SC network was significantly different among the three groups but not vice versa (Fig. 3a, b). Overall, these results demonstrated that the abnormal SC profiles involved in suicide attempters with BD depression had direct impacts on the altered FC profiles.

Several limitations should be noted. First, although there was no use of psychotropic medications in the previous 6 weeks before the neuroimaging scanning, it may take longer for patients to stabilize after changing or stopping the medication. Second, structural and functional coupling was only assessed for anatomically connected regions, while FC could also result from indirect pathways [15]. Move advanced computational methods are needed to consider the effect of these pathways on FC. In addition, the averaged FC over the entire recording, whereas FC has been indicated to possess dynamic property [54-57]. Even though the dynamic of FC is not the scope of the current study, this question remains to be essential for future understanding of the pathology. Moreover, we did not differentiate between BD-I and BD-II groups, while there might be subtle differences in BD subtypes. Besides, our findings were specific to BD patients with a current major depressive episode and not necessarily extended to BD patients with a manic episode or mixed episode. Lastly, our study was a cross-session design; longitudinal studies are needed to fully investigate the development of system features, their transition from suicidal ideation to suicidal behavior in $B D$, and whether it is generalized to other disorders.

In summary, we demonstrated altered SC and FC networks along with SC-FC decoupling in BD depression patients with suicide attempts compared with non-attempters. Critically, the 
altered SC network constrains the abnormal FC network profile, suggesting the structural connectome is the key determinant of functional connectome dysfunction. Furthermore, SC-FC decoupling in the altered SC and FC networks was correlated with suicide risk, indicating it is a more sensitive maker than single SC or FC modality. Overall, our findings may shed new light on the pathology of suicide behaviors and have implications for early intervention and prevention strategies.

\section{FUNDING AND DISCLOSURE}

This work was supported by the grants of National Key R\&D Program of China (2018YFC1314601); the National Natural Science Foundation of China (81701784, 81871066); Jiangsu Provincial Medical Innovation Team of the Project of Invigorating Health Care through Science, Technology and Education (CXTDC2016004); Jiangsu Provincial key research and development program (BE2018609, BE2019675); Key Project supported by Medical Science and Technology Development Foundation, Jiangsu Commission of Health (K2019011); Key Project supported by Medical Science and Technology Development Foundation, Nanjing Department of Health (YKK16146, YKK17135, ZKX18034); Science and Technology Program of Nanjing (201605039); Nanjing Medical University Science and Technology Development Project (NMUB2018204); Youth Medical Talent Project of Jiangsu Province (QNRC2016050). The authors declare no competing interests.

\section{ACKNOWLEDGEMENTS}

We thank all participants and staffs of the Affiliated Brain Hospital of Nanjing Medical University for their contributions to data generation.

\section{AUTHOR CONTRIBUTIONS}

$\mathrm{HJ}, \mathrm{RZ}, \mathrm{ZY}$, and QL conceived and designed this research. HJ, RZ, ST, HW, ZC, XW, JS, $J Q, J S, H L$, and YC performed research, HJ, RZ, ST, and HW analyzed data. HJ, RZ, ZY, QL wrote the paper. All authors reviewed the final submission and gave final approval of the submitted version.

\section{ADDITIONAL INFORMATION}

Supplementary Information accompanies this paper at (https://doi.org/10.1038/ s41386-020-0753-5).

Publisher's note Springer Nature remains neutral with regard to jurisdictional claims in published maps and institutional affiliations.

\section{REFERENCES}

1. Plans L, Barrot C, Nieto E, Rios J, Schulze TG, Papiol S, et al. Association between completed suicide and bipolar disorder: a systematic review of the literature. J Affect Disord. 2019;242:111-22.

2. Pompili $M$, Gonda $X$, Serafini $G$, Innamorati $M$, Sher $L$, Amore $M$, et al. Epidemiology of suicide in bipolar disorders: a systematic review of the literature. Bipolar Disord. 2013;15:457-90.

3. Novick DM, Swartz HA, Frank E. Suicide attempts in bipolar I and bipolar II disorder: a review and meta-analysis of the evidence. Bipolar Disord. 2010;12:1-9.

4. Gosnell SN, Fowler JC, Salas R. Classifying suicidal behavior with resting-state functional connectivity and structural neuroimaging. Acta Psychiatr Scand. 2019;140:20-9.

5. Hagmann P, Cammoun L, Gigandet X, Meuli R, Honey CJ, Wedeen VJ, et al. Mapping the structural core of human cerebral cortex. PLoS Biol. 2008;6:e159.

6. Geng J, Yan R, Shi J, Chen Y, Mo Z, Shao J, et al. Altered regional homogeneity in patients with somatic depression: a resting-state fMRI study. J Affect Disord. 2019;246:498-505.

7. Lu Q, Jiang HT, Liu HY, Liu G, Teng GJ, Yao ZJ. Depression severity evaluation for female patients based on a functional mri model. J Magn Reson Imaging. 2010;31:1067-74.

8. Tian S, Zhang S, Mo Z, Chattun MR, Wang Q, Wang L, et al. Antidepressants normalize brain flexibility associated with multi-dimensional symptoms in major depressive patients. Prog Neuropsychopharmacol Biol Psychiatry. 2020;100:109866.
9. Ding Y, Pereira F, Hoehne A, Beaulieu MM, Lepage M, Turecki G, et al. Altered brain processing of decision-making in healthy first-degree biological relatives of suicide completers. Mol Psychiatry. 2017;22:1149-54.

10. Johnston JAY, Wang F, Liu J, Blond BN, Wallace A, Liu J, et al. Multimodal neuroimaging of frontolimbic structure and function associated with suicide attempts in adolescents and young adults with bipolar disorder. Am J Psychiatry. 2017;174:667-75.

11. Pandey GN, Dwivedi Y, Ren X, Rizavi HS, Mondal AC, Shukla PK, et al. Brain region specific alterations in the protein and mRNA levels of protein kinase $A$ subunits in the post-mortem brain of teenage suicide victims. Neuropsychopharmacology. 2005;30:1548-56.

12. Lewis CP, Nakonezny PA, Blacker CJ, Vande Voort JL, Port JD, Worrell GA, et al. Cortical inhibitory markers of lifetime suicidal behavior in depressed adolescents. Neuropsychopharmacology. 2018;43:1822-31.

13. Zhang RB, Shao ROB, Xu GY, Lu WC, Zheng WJ, Miao QZ, et al. Aberrant brain structural-functional connectivity coupling in euthymic bipolar disorder. Hum Brain Mapp. 2019;40:3452-63.

14. Zhang ZQ, Liao W, Chen HF, Mantini D, Ding JR, Xu Q, et al. Altered functionalstructural coupling of large-scale brain networks in idiopathic generalized epilepsy. Brain. 2011;134:2912-28.

15. Honey CJ, Sporns O, Cammoun L, Gigandet X, Thiran JP, Meuli R, et al. Predicting human resting-state functional connectivity from structural connectivity. Proc Natl Acad Sci USA. 2009;106:2035-40.

16. Dai Z, Lin Q, Li T, Wang X, Yuan H, Yu X, et al. Disrupted structural and functional brain networks in Alzheimer's disease. Neurobiol Aging. 2019;75:71-82.

17. Zhang J, Zhang Y, Wang L, Sang L, Yang J, Yan R, et al. Disrupted structural and functional connectivity networks in ischemic stroke patients. Neuroscience. 2017;364:212-25.

18. Jiang $H$, Bahramisharif $A$, van Gerven MAJ, Jensen O. Distinct directional couplings between slow and fast gamma power to the phase of theta oscillations in the rat hippocampus. Eur J Neurosci. 2020;51:2070-81.

19. Sheehan DV, Lecrubier $Y$, Sheehan $\mathrm{KH}$, Amorim $\mathrm{P}$, Janavs J, Weiller $E$, et al. The Mini-International Neuropsychiatric Interview (M.I.N.I.): the development and validation of a structured diagnostic psychiatric interview for DSM-IV and ICD-10. J Clin Psychiatry. 1998:59:22-33.

20. Lish JD, Weissman MM, Adams PB, Hoven CW, Bird H. Family psychiatric screening instruments for epidemiologic studies: pilot testing and validation. Psychiatry Res. 1995;57:169-80.

21. Monkul ES, Hatch JP, Nicoletti MA, Spence $S$, Brambilla $P$, Lacerda $A L$, et al. Fronto-limbic brain structures in suicidal and non-suicidal female patients with major depressive disorder. Mol Psychiatry. 2007;12:360-6.

22. World Medical A. World Medical Association Declaration of Helsinki: ethical principles for medical research involving human subjects. JAMA. 2013;310: 2191-4.

23. Hamilton M. A rating scale for depression. J Neurol Neurosurg Psychiatry. 1960; 23:56-62.

24. Hamilton M. The assessment of anxiety states by rating. $\mathrm{Br} J$ Med Psychol. 1959;32:50-5.

25. Cutcliffe JR, Barker P. The Nurses' Global Assessment of Suicide Risk (NGASR): developing a tool for clinical practice. J Psychiatr Ment Health Nurs. 2004;11: 393-400.

26. Jenkinson M, Beckmann CF, Behrens TE, Woolrich MW, Smith SM. Fsl. NeuroImage. 2012;62:782-90.

27. Xie S, Chen L, Zuo N, Jiang T. DiffusionKit: A light one-stop solution for diffusion MRI data analysis. J Neurosci Methods. 2016;273:107-19.

28. Mori S, Crain BJ, Chacko VP, van Zijl PC. Three-dimensional tracking of axonal projections in the brain by magnetic resonance imaging. Ann Neurol. 1999; 45:265-9.

29. Chao-Gan Y, Yu-Feng Z. DPARSF: a MATLAB toolbox for "pipeline" data analysis of resting-state fmri. Front Syst Neurosci. 2010;4:13.

30. Tzourio-Mazoyer N, Landeau B, Papathanassiou D, Crivello F, Etard O, Delcroix N, et al. Automated anatomical labeling of activations in SPM using a macroscopic anatomical parcellation of the MNI MRI single-subject brain. Neurolmage. 2002;15:273-89.

31. Zalesky A, Fornito A, Bullmore ET. Network-based statistic: identifying differences in brain networks. Neurolmage. 2010;53:1197-207.

32. Chung SJ, Kim HR, Jung JH, Lee PH, Jeong Y, Sohn YH. Identifying the functional brain network of motor reserve in early Parkinson's disease. Mov Disord. 2020;35:577-86.

33. Chahal R, Gotlib IH, Guyer AE. Research review: brain network connectivity and the heterogeneity of depression in adolescence-a precision mental health perspective. J Child Psychol Psychiatry. 2020. https://doi.org/10.1111/jcpp.13250.

34. Perlis RH, Ostacher MJ, Goldberg JF, Miklowitz DJ, Friedman E, Calabrese J, et al. Transition to mania during treatment of bipolar depression. Neuropsychopharmacology. 2010;35:2545-52. 
35. Auerbach RP, Pisoni A, Bondy E, Kumar P, Stewart JG, Yendiki A, et al. Neuroanatomical prediction of anhedonia in adolescents. Neuropsychopharmacology. 2017;42:2087-95.

36. Brown VM, Wilson J, Hallquist MN, Szanto K, Dombrovski AY. Ventromedial prefrontal value signals and functional connectivity during decision-making in suicidal behavior and impulsivity. Neuropsychopharmacology. 2020;45: 1034-41.

37. Schmaal L, van Harmelen AL, Chatzi V, Lippard ETC, Toenders YJ, Averill LA, et al. Imaging suicidal thoughts and behaviors: a comprehensive review of 2 decades of neuroimaging studies. Mol Psychiatry. 2020;25:408-27.

38. Tanaka SC, Doya K, Okada G, Ueda K, Okamoto Y, Yamawaki S. Prediction of immediate and future rewards differentially recruits cortico-basal ganglia loops. Nat Neurosci. 2004;7:887-93.

39. van Heeringen $K$, Van den Abbeele D, Vervaet M, Soenen L, Audenaert K. The functional neuroanatomy of mental pain in depression. Psychiatry Res. 2010; 181:141-4.

40. Jollant F, Lawrence NS, Giampietro V, Brammer MJ, Fullana MA, Drapier D, et al. Orbitofrontal cortex response to angry faces in men with histories of suicide attempts. Am J Psychiatry. 2008;165:740-8.

41. van Heeringen K, Mann JJ. The neurobiology of suicide. Lancet Psychiatry. 2014; 1:63-72.

42. Bari A, Robbins TW. Inhibition and impulsivity: behavioral and neural basis of response control. Prog Neurobiol. 2013;108:44-79.

43. Mann JJ. Neurobiology of suicidal behaviour. Nat Rev Neurosci. 2003;4:819-28.

44. Pan LA, Hassel S, Segreti AM, Nau SA, Brent DA, Phillips ML. Differential patterns of activity and functional connectivity in emotion processing neural circuitry to angry and happy faces in adolescents with and without suicide attempt. Psychol Med. 2013;43:2129-42.

45. Reisch T, Seifritz E, Esposito F, Wiest R, Valach L, Michel K. An fMRI study on mental pain and suicidal behavior. J Affect Disord. 2010;126:321-5.

46. Torres-Platas SG, Hercher C, Davoli MA, Maussion G, Labonte B, Turecki G, et al. Astrocytic hypertrophy in anterior cingulate white matter of depressed suicides. Neuropsychopharmacology. 2011;36:2650-8.
47. Jung J, Choi S, Han KM, Kim A, Kang W, Paik JW, et al. Alterations in functional brain networks in depressed patients with a suicide attempt history. Neuropsychopharmacology. 2020;45:964-74.

48. Supekar K, Uddin LQ, Prater K, Amin H, Greicius MD, Menon V. Development of functional and structural connectivity within the default mode network in young children. Neurolmage. 2010;52:290-301.

49. Hagmann P, Sporns O, Madan N, Cammoun L, Pienaar R, Wedeen VJ, et al. White matter maturation reshapes structural connectivity in the late developing human brain. Proc Natl Acad Sci USA. 2010;107:19067-72.

50. Gamboa OL, Tagliazucchi E, von Wegner F, Jurcoane A, Wahl M, Laufs $H$, et al. Working memory performance of early MS patients correlates inversely with modularity increases in resting state functional connectivity networks. NeuroImage. 2014;94:385-95.

51. Jiang H, Dai Z, Lu Q, Yao Z. Magnetoencephalography resting-state spectral fingerprints distinguish bipolar depression and unipolar depression. Bipolar Disord. 2019;00:1-9. https://doi.org/10.1111/bdi.12871.

52. Jiang $\mathrm{H}$, Tian $\mathrm{S}, \mathrm{Bi} K$, Lu $\mathrm{Q}$, Yao $\mathrm{Z}$. Hyperactive frontolimbic and frontocentral resting-state gamma connectivity in major depressive disorder. J Affect Disord. 2019;257:74-82.

53. Melozzi F, Bergmann E, Harris JA, Kahn I, Jirsa V, Bernard C. Individual structural features constrain the mouse functional connectome. Proc Natl Acad Sci USA. 2019;116:26961-69.

54. Shen K, Misic B, Cipollini BN, Bezgin G, Buschkuehl M, Hutchison RM, et al. Stable long-range interhemispheric coordination is supported by direct anatomical projections. Proc Natl Acad Sci USA. 2015;112:6473-8.

55. Tian S, Chattun MR, Zhang S, Bi K, Tang H, Yan R, et al. Dynamic community structure in major depressive disorder: a resting-state MEG study. Prog Neuropsychopharmacol Biol Psychiatry. 2019;92:39-47.

56. Lu Q, Jiang H, Luo G, Han Y, Yao Z. Multichannel matching pursuit of MEG signals for discriminative oscillation pattern detection in depression. Int J Psychophysiol. 2013;88:206-12.

57. Lu Q, Jiang H, Bi K, Liu C, Yao Z. Discriminative analysis with a limited number of MEG trials in depression. J Affect Disord. 2014;167:207-14. 